\title{
基于生态位模型预测天麻全球潜在适生区
}

\author{
张 琴 ${ }^{1}$ 张东方 ${ }^{1,2}$ 吴明丽 ${ }^{3}$ 郭 杰 $^{1,4}$ 孙成忠 ${ }^{5}$ 谢彩香 $^{*}$
}

${ }^{1}$ 中国医学科学院北京协和医学院药用植物研究所, 北京 100193 ; ${ }^{2}$ 江苏师范大学地理测绘与城乡规划学院, 江苏徐州 221116 ; ${ }^{3}$ 湖北中医药大学药学 院, 武汉 430065 ; $^{4}$ 武汉理工大学化学化工与生命科学学院, 武汉 $430070{ }^{5}$ 中国测绘科学研究院, 北京 100039

摘 要 目前对药用植物天麻(Gastrodia elata)的全球潜在适生区研究较少, 基于多个生态位模型预测天麻在全球范围内的潜 在适生区，对天麻人工引种栽培及其产业发展具有重要意义。该文收集 220 个天麻全球分布点和 19 个生态因子数据，最终筛选 出 8 个环境变量参与模型训练, 基于 3 个生态位模型(BIOCLIM、DOMAIN和MAXENT)预测天麻全球潜在适生区, 并采用受试 者工作特征曲线ROC和Kappa统计量分析比较不同模型的预测效果。结果表明: 3 个模型的预测结果基本一致, 天麻全球潜在 适生区主要分布在 $20^{\circ}-50^{\circ} \mathrm{N}$ 的亚洲地区, 其中中国、日本和韩国是集中分布地, 此外, 印度、尼泊尔以及欧洲地中海附近有 少量适生区。其中最适宜区主要分布在: 中国四川盆地附近的省区以及中东部; 韩国中东部的忠清北道、庆尚北道和庆尚南 道; 日本本州岛、九州岛以及四国岛, 因此中国、日本和韩国是天麻的主要产区。3个模型的受试者工作特征曲线下面积(AUC 值)平均值均达到 0.9 以上, Kappa平均值均达到 0.65 以上, 能较好地预测天麻的潜在适生区, 其中MAXENT模型的精度较高, 其次是DOMAIN和BIOCLIM模型。

关键词 天麻; 生态位模型; 潜在适生区; 模型评价

引用格式: 张琴, 张东方, 吴明丽, 郭杰, 孙成忠, 谢彩香 (2017). 基于生态位模型预测天麻全球潜在适生区. 植物生态学报, 41, 770-778. doi: $10.17521 /$ cjpe. 2016.0380

\section{Predicting the global areas for potential distribution of Gastrodia elata based on ecological niche models}

ZHANG Qin ${ }^{1}$, ZHANG Dong-Fang ${ }^{1,2}$, WU Ming-Li ${ }^{3}$, GUO Jie ${ }^{1,4}$, SUN Cheng-Zhong ${ }^{5}$, and XIE Cai-Xiang ${ }^{1 *}$

${ }^{1}$ Institute of Medicinal Plant Development, Peking Union Medical College, Chinese Academy of Medical Sciences, Beijing 100193, China; ${ }^{2}$ School of Geographical Surveying and Urban and Rural Planning, Jiangsu Normal University, Xuzhou, Jiangsu 221116, China; ${ }^{3}$ Pharmacy Faculty, Hubei University of Chinese Medicine, Wuhan 430065, China; ${ }^{4}$ School of Chemistry, Chemical Engineering and Life Sciences, Wuhan University of Technology, Wuhan 430070, China; and ${ }^{5}$ Chinese Academy of Surveying \& Mapping, Beijing 100039, China

\section{Abstract}

Aims Previous studies on the globally suitable areas for growing the medicinal plant Gastrodia elata is lacking. This study aims to predict the global areas for potential distribution of this plant based on multiple ecological niche models.

Methods A total of 220 global distribution points of G. elata and 19 ecological variables were compiled and eight environmental variables were selected for the model training. Three ecological niche models, including BIOCLIM, DOMAIN, and MAXENT, were used to predict the global areas for potential distribution of G. elata. The resulting data of different models were analyzed and compared with two statistical criteria: the area under the receiver operating characteristic curve (AUC) and Kappa value.

Important findings The predictions of the three models are basically identical, showing that the global areas for potential distribution of G. elata are predominantly in the range of $20^{\circ} \mathrm{N}$ to $50^{\circ} \mathrm{N}$ in Asia, mainly in China, South Korea and Japan. A small proportion of the suitable areas occur in India, Nepal and the European countries near Mediterranean. The most suitable areas distribute in provinces close to the Sichuan Basin and the central East China, the mid-eastern parts of South Korea such as Chungcheongbuk-do, Gyeongsangbuk-do and Gyeongsangnamdo, and the Kyushu region and the Shikoku region on Japan's main island. Therefore, these three countries can be used as the main production areas of G. elata for its commercial development. The AUC average values of the three models are all above 0.9 and the Kappa average values all above 0.65 , justifying their applications for predicting the potential areas of G. elata. Among them, the MAXENT model appears to perform the best, followed by

收稿日期Received: 2016-12-12 接受日期Accepted: 2017-05-31

* 通信作者Author for correspondence (E-mail: caixiangxie@163.com) 
DOMAIN and BIOCLIM.

Key words Gastrodia elata; ecological niche models; potential suitable distribution area; model evaluation

Citation: Zhang Q, Zhang DF, Wu ML, Guo J, Sun CZ, Xie CX (2017). Predicting the global areas for potential distribution of Gastrodia elata based on ecological niche models. Chinese Journal of Plant Ecology, 41, 770-778. doi: 10.17521/cjpe.2016.0380

天麻(Gastrodia elata)为兰科(Orchidaceae)天麻 属植物, 又名赤箭、独摇芝、定风草、离母。其块 茎是一种名贵中药, 具有息风止痉、平抑肝阳、祛 风通络的功效，常用于治疗头晕目眩、肢体麻木、 失眠、中风、癫㾁等症(陶云海, 2008; 柴锟等, 2014; 国家药典委员会, 2015)。天麻也是深受现代人青睐 的保健食材, 在调理和增强人体机能方面发挥着重 要作用。随着对天麻有效成分及其药理作用的深入 研究, 天麻的临床需求量以及保健品用量也在不断 增加。目前, 中国天麻的年出口量约500吨, 国内的 年需求量约2万吨(胡一冰等, 2001; Kim et al., 2003; 张金霞等, 2013)。

天麻是一种无根无叶的异养兰科植物, 其种子 萌发后主要依靠分解侵入其体内的蜜环菌菌丝获得 营养。天麻及其共生菌主要分布于亚热带、温带及 寒温带的山地, 主要产自亚洲东部的中国、韩国、 日本和俄罗斯远东地区, 产量以中国最多。野生天 麻品质较好, 但是多年来遭到过度采挖, 其资源日 见枯竭, 如今市场上的天麻多来源于人工栽培。目 前在天麻栽培生产过程中仍存在许多问题, 如天 麻、萌发菌和蜜环菌品种退化, 栽培密集导致减产, 菌材利用种类少, 种群种类失衡等, 导致天麻的产 量远远跟不上市场需求, 严重影响天麻的产业发展 (王丽馥和周常霞, 2013; 徐锦堂, 2013; 胡荣丽, 2015)。近年来有很多国内外研究均表明环境因子对 植物生长和分布具有重要影响, 如土壤条件影响植 物的矿质元素含量、水分吸收, 土壤中微生物也会 影响植物的生长及分布(Engler et al., 2011; 邵璞和 曾晓东, 2011)。天麻属异养植物, 其生长依赖于蜜 环菌菌丝提供营养, 但是蜜环菌的生长也会受到环 境因子(如温度和湿度)的影响(孙立夫等, 2007), 因 此环境可通过影响蜜环菌生长进而影响天麻生长。 天麻的分布与主产区的形成是生态因子综合作用于 天麻和蜜环菌的结果(彭华胜和王德群, 2007), 适合 天麻生长的地区也适合蜜环菌生长, 所以环境作为 生物赖以生存的媒介, 是影响天麻生长分布的主要 因素。目前关于天麻的研究多侧重于其种质资源的
遗传多样性、栽培技术、药理药化分析等方面, 对 天麻的栽培选址研究相对较少。因此, 为了避免盲 目引种扩种带来的经济损失, 通过现代研究方法预 测天麻全球潜在适生区，扩大天麻栽培面积，提高 天麻产量和质量, 是解决天麻产业发展问题的一种 有效途径。

生态位模型是利用物种已知的分布数据和相 关环境变量, 根据一定的算法运算来构建模型, 判 断物种的生态需求, 并将运算结果投射至不同的时 间和空间中以预测物种的实际分布和潜在分布 (Kulhanek et al., 2011)。目前生态位模型种类很多, 本文采用常用的 3 个模型(BIOCLIM、DOMAIN和 MAXENT)模拟天麻全球潜在适生区。BIOCLIM模 型利用已知的物种分布资料和环境数据产生以生态 位为基础的物种生态需求，探索物种已知分布区的 环境特征与研究区域的非随机关系(Busby, 1991); DOMAIN模型利用Gower算法，通过点和点之间的 相似矩阵来计算目标点上环境变量的适宜程度, 最 终确定物种的分布范围(Carpenter et al., 1993); MAXENT模型是将物种与其生长环境视为一个系 统, 通过计算系统具有最大熵时的状态参数确定物 种和环境之间的稳定关系, 以此估计物种的地理分 布(Phillips et al., 2006)。目前用生态位模型预测天麻 潜在适生区的研究仅限于单个模型及小范围区域, 用多个模型预测天麻全球潜在适生区的研究尚未见 报道。本文通过数据库和文献检索, 收集天麻全球 分布数据, 以 19 个气候因子为环境数据, 采用 3 个模 型预测天麻全球潜在适生区，比较不同模型的预测 结果, 分析全球范围内适合天麻生产的主要区域, 旨在为天麻科学引种栽培提供参考, 提高天麻产量 和质量。

\section{1 材料和方法}

\section{1 材料}

\subsection{1 天麻全球样本点}

根据全球生物多样性信息数据库网络(GBIF, http://www.gbif.org/), 中国数字植物标本馆 $(\mathrm{CVH}$, 
http://www.cvh.org.cn/), The plant list (http://www. theplantlist.org/) 以及文献检索得到全球天麻样本点。 为保证天麻样本点信息准确, 对所获得的样本点进 行严格笁选。首先, 选取拉丁名准确和经纬度信息 详实的点, 剔除拉丁名错误和经纬度信息不全以及 重复的点; 其次, 对于有确切地名而无经纬度的点, 进一步根据“谷歌地球”进行经纬度定位，最终得到 220 个天麻样本点, 并将天麻的分布数据保存为 shp 和csv格式。样本年份范围为1930-2008年, 主要分 布在 $20^{\circ}-50^{\circ} \mathrm{N}, 80^{\circ}-160^{\circ} \mathrm{E}$, 其中中国、日本和韩国 分布较为集中, 俄罗斯、印度、尼泊尔等地有少数 分布点。

\subsection{2 环境数据}

在大尺度空间影响物种分布的环境变量主要 是气候因素(朱耿平等, 2013), 因此本文采用与温度 和降水相关的 19 个气候变量, 见表 1 , 其来源于世界 气候数据库 (http://www.worldclim.org//)1960-1990 年监测数据的平均值, 数据分辨率为 $2.5 \mathrm{~min}$ 。地图 资料来源于国家基础地理信息数据库 (http://www.sbsm.gov.cn/)。

\begin{tabular}{ll} 
表1 气候变量 & \\
Table 1 Climatic variables & \\
\hline 气候变量 Climatic variables & 单位 Unit \\
\hline 年平均温度 Annual mean temperature & ${ }^{\circ} \mathrm{C}$ \\
月平均温度范围 Mean monthly temperature range & ${ }^{\circ} \mathrm{C}$ \\
等温性 Isothermality & - \\
温度的季节性 Temperature seasonality & - \\
最热月份最高温度 Max temperature of warmest month & ${ }^{\circ} \mathrm{C}$ \\
最冷月份最低温度 Min temperature of coldest month & ${ }^{\circ} \mathrm{C}$ \\
年温度变化范围 Annual temperature range & ${ }^{\circ} \mathrm{C}$ \\
最湿季平均温度 Mean temperature of wettest quarter & ${ }^{\circ} \mathrm{C}$ \\
最干季平均温度 Mean temperature of driest quarter & ${ }^{\circ} \mathrm{C}$ \\
最暖季平均温度 Mean temperature of warmest quarter & ${ }^{\circ} \mathrm{C}$ \\
最冷季平均温度 Mean temperature of coldest quarter & ${ }^{\circ} \mathrm{C}$ \\
年降水量 Annual precipitation & $\mathrm{mm}$ \\
最湿月份降水量 Precipitation of wettest month & $\mathrm{mm}$ \\
最干月份降水量 Precipitation of driest month & $\mathrm{mm}$ \\
降水的季节性 Precipitation seasonality & $\mathrm{mm}$ \\
最湿季降水量 Precipitation of wettest quarter & - \\
最干季降水量 Precipitation of driest quarter & $\mathrm{mm}$ \\
最暖季降水量 Precipitation of warmest quarter \\
最冷季降水量 Precipitation of coldest quarter \\
\hline 气候变量来源于世界气候数据库(http://www.worldclim.org//)。-, 无单 \\
位。 \\
Climatic variables are from Global Climate Data (http://www.worldclim.org//). \\
-, without unit. & $\mathrm{mm}$ \\
Ww.plant-ecology.com & $\mathrm{mm}$ \\
\hline
\end{tabular}

\subsection{3 生态位模型}

DIVA-GIS 软件(Version 7.5)下载自http:// www.diva-gis.org/download, 用于天麻分布数据集 的采样以及 BIOCLIM 和 DOMAIN 模型分析。 MAXENT模型软件 (Version 3.3.3k)下载自 http:// www.cs.princeton.edu/ schapire/maxent.，用于最大 熵模型预测。ArcGIS 10.1是由ESRI公司开发的一个 全面的地理信息系统平台, 用于图层和数据的格式 转换以及重分类等。

\section{2 方法}

\subsection{1 样本数据处理}

在DIVA-GIS软件中, 利用 Sample Points工具, 设置 $75 \%$ 的已知分布点集, 剩下的 $25 \%$ 和随机选取 10 倍于分布点的背景点作为测试样本, 为比较 3 种 模型预测结果的差异性, 随机生成 10 组训练数据集 和对应的测试数据集, 训练集用于模型预测, 测试 集用于模型验证。

\subsection{2 环境数据共线性诊断}

为了避免生态因子之间的共线性造成模型预 测结果过度拟合(Graham, 2003), 用Spearman系数 法对以上环境变量进行相关性检验，保留Spearman 系数小于 0.75 的变量, 对于 Spearman系数大于 0.75 的两个相关性变量则选择其中一个生态意义重大的 因子(Zhang et al., 2016), 最终得到 8 个气候变量, 分 别为年平均温度、月平均温度范围、等温性、温度 的季节性、最热月份最高温度、年降水量、最湿月 份降水量、降水的季节性。

\subsection{3 模型预测方法}

MAXENT模型：导入 10 组 $\operatorname{csv}$ 格式的训练数据 集和asc格式环境数据集，设置刀切法权重，运算参 数、运算规则等采用默认设置, 预测结果图以asc格 式输出。

BIOCLIM模型和DOMAIN模型：在DIVA-GIS 中首先添加 shp格式的训练数据集, 再将 8 个气候数 据图层转换成grd格式, 再生成stack数据集。然后, 在Modeling-Bioclim/Domain模块中添加stack格式的 环境数据集, 依次进行BIOCLIM 和DOMAIN模型 的预测(王运生, 2007)。

\subsection{4 模型预测准确性评估}

采用受试者工作特征曲线(ROC)和Kappa统计 量以验证模型预测精度。将各个模型的预测图在 DIVA-GIS中转换成 grd格式, 并建立相应的stack数 
据集, 再导入验证点图层, 创建评估文件, 并输出 $\mathrm{ROC}$ 和Kappa值。

$\mathrm{ROC}$ 曲线分析法在物种潜在分布预测模型评 价中得到了广泛的应用, 是目前认可度较高的诊断 试验评价指标(Peterson et al., 2007)。ROC曲线下的 面积即为AUC值 (area under curve), AUC的数值范围 为 $0.5-1.0$, 值越大表示预测越精确(Swets, 1988)。 Kappa统计量是一种一致性检验方法, 广泛用于模 型评价, 其综合考虑了物种分布率、灵敏度和特异 度, 取值范围为 $[-1,+1]$, 当Kappa统计量为 1 时表示 预测模型理想，等于或小于 0 时表示模型预测效果 没有随机模型好(Manel et al., 2001; Segurado \& Araújo, 2004), 当Kappa值大于 0.6 时, 一致性显著, 值越大，预测精度越高(许文宁等, 2011)。

\section{2 结果}

\section{1 天麻全球潜在适生区}

从每个模型的 10 组预测图中选出 AUC最大的 图作为底图, 将其转换成ASCII格式导入 GIS, 进行 栅格重分类，根据不同模型预测结果的默认值，利 用自然间断点分级法(Jenks)对物种适生区进行等级 划分, 得到各个模型的适生区分布图, 如图1所示。

图1A所示为BIOCLIM模型预测结果, 结果显 示天麻潜在适生区主要分布在 $20^{\circ}-50^{\circ} \mathrm{N}$ 的亚洲地 区, 主要集中在中国、韩国和日本, 此外, 印度、尼 泊尔、巴基斯坦、阿富汗、土耳其、希腊、法国和 地中海附近地区, 以及北美洲美国中部和大西洋附 近地区也有少量适生区。中国的天麻潜在适生区主 要位于四川盆地附近的省区以及中东部, 韩国和日 本的潜在适生区覆盖度较大, 包括其国土的大部分 地区。其中最适宜区主要分布在: 中国四川、贵州、 湖南、湖北省西部, 陕西和四川交界处, 河南西部, 安徽、江苏等省份; 韩国庆尚北道、庆尚南道以及 全罗北道和全罗南道, 如清道郡、青松郡、蔚州郡、 密阳市、梁山市、金海市、昌宁郡、宜宁郡、谷城 郡、求礼郡、顺天市、长兴郡、康津郡、珍岛郡等; 日本的本岛地区以及四国地区，如琦玉县、山梨县、 东京都、静冈县、神奈川县、山口县、爱媛县、山 口县等。图1B是DOMAIN模型预测结果, 天麻在全 球范围内潜在适生区集中分布于中国、韩国、日本 和俄罗斯, 此外, 蒙古和印度有部分适生区。其中最 适宜区主要分布于: 中国云南、贵州、四川、湖南、
湖北、陕西、江西、安徽、江苏、浙江、山西、内 蒙、辽宁、吉林; 韩国忠清北道、忠清南道、全罗 北道、全罗南道、庆尚北道西部; 日本本岛地区，如 岛根县、广岛县、山口县、和歌山县、长崎县、青 森县、秋田县、福冈县、佐贺县; 俄罗斯远东部分 地区, 如西伯利亚北部及东北部。图1C为MAXENT 模型预测结果，据图可知：天麻的潜在分布区主要 位于北半球的亚洲地区, 主要集中在中国、韩国和 日本，此外朝鲜、印度、尼泊尔、巴基斯坦、阿富 汗、土库曼斯坦以及欧洲地中海附近地区有少量适 生区。其中最适宜区主要分布于: 中国四川盆地附 近的省区以及中国中东部地区，如四川省、贵州省、 云南省昭通地区、重庆市、湖南省、湖北省、陕西 省与四川省交界处、河南省西部、安徽省、浙江省、 江西省; 韩国的绝大部分地区，如庆尚南道、庆尚 北道, 全罗北道、全罗南道、忠清南道、忠清北道 等; 日本的本州岛、九州岛以及四国岛地区。

\section{2 不同模型预测准确性评估}

各个模型的AUC值和Kappa值如表2所示。分析 表2可知, MAXENT模型AUC平均值为 0.980 , BIOCLIM 模型 $\mathrm{AUC}$ 平均值为 0.936 , DOMAIN 模型的 AUC平均值为 $0.960,3$ 种模型的AUC平均值均达到 0.9 以上, 远远超过随机模型 $(\mathrm{AUC}=0.5)$, 表明 3 种 模型对天麻均有较好的预测效果, 其中MAXENT 模型的AUC平均值最大。3种模型AUC值的标准偏 差大小依次为BIOCLIM $>$ DOMAIN $>$ MAXENT, 对3种模型的AUC平均值进行单因素方差分析, 各 模型两两之间差异显著 $(p<0.05)$, 各模型AUC值的 离散程度, MAXENT 最小, 其次是 DOMAIN 和 BIOCLIM。因此综合比较, 3种模型的总体预测水平 以MAXENT为最高, 其次是DOMAIN和BIOCLIM。

分析3 个模型的Kappa值可知, MAXENT模型的 Kappa平均值为 0.694 , DOMAIN和BIOCLIM模型的 Kappa平均值分别为 0.791 和 0.837 , 表明 3 种模型一 致性显著，预测效果均达到良好以上，可用于预测 天麻全球潜在适生区。从图2可知, DOMAIN和 BIOCLIM模型的Kappa平均值和AUC平均值具有较 好的一致性, AUC平均值较大, Kappa平均值也较大, 而MAXENT模型的AUC平均值高于另外两个模型, Kappa平均值小于这两个模型。单从Kappa统计量分 析，则对天麻而言，DOMAIN模型也可作为预测其 分布的最佳模型。 

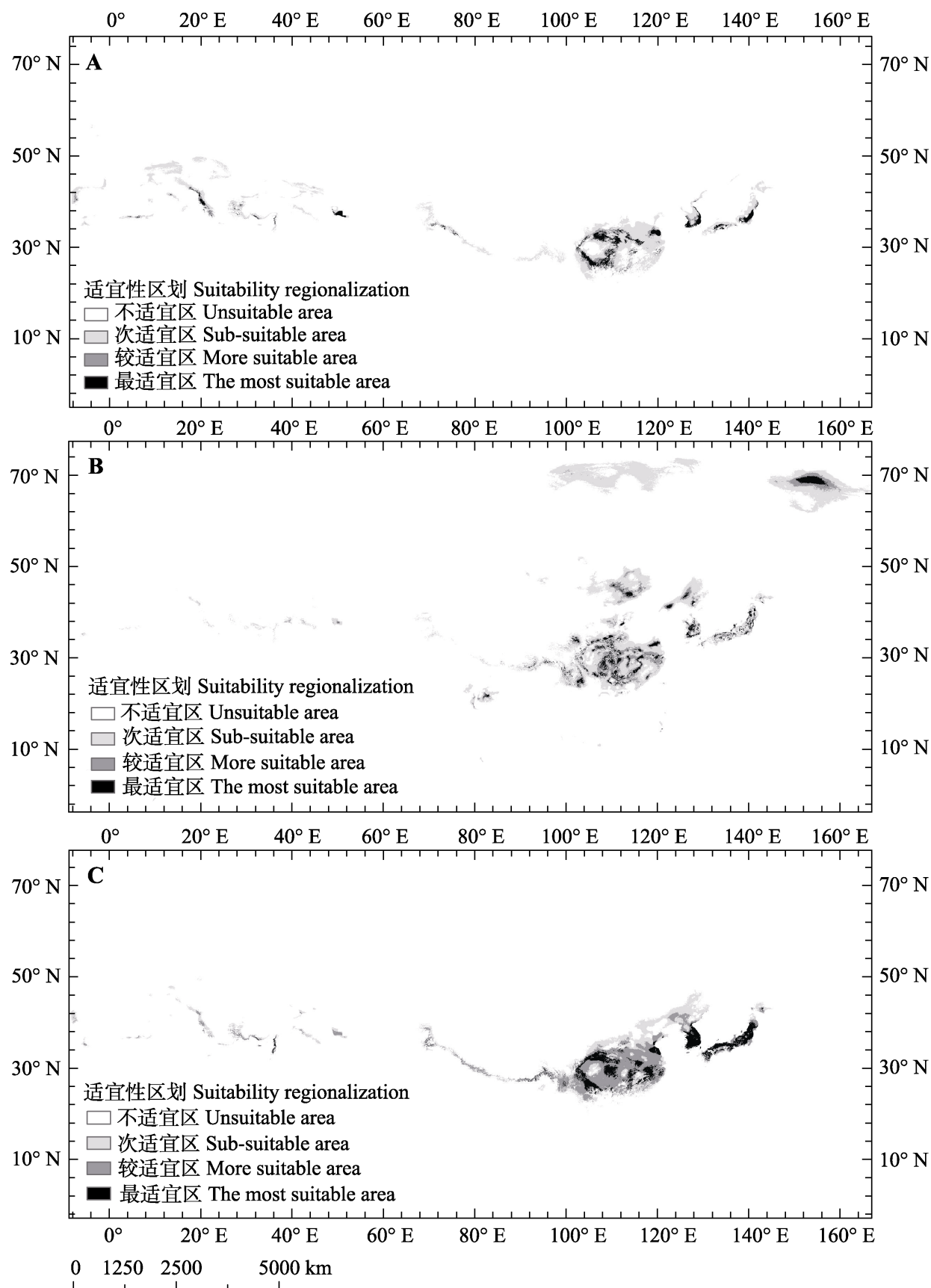

图1 不同生态位模型预测天麻全球潜在适生区。A, BIOCLIM模型。B, DOMAIN模型。C, MAXENT模型。

Fig. 1 Global areas for potential distribution of Gastrodia elata based on different ecological niche models. A, Based on BIOCLIM. B, Based on DOAMIN. C, Based on MAXENT.

\section{3 讨论}

应用生态位模型预测物种潜在适生区的前提 是假设物种的生态位需求保守且物种的生态需求和 分布处于平衡的状态(Peterson et al., 2011), 植物的 迁移较缓慢, 生态位漂移不明显, 在短期内物种分 布数据和环境数据变化不大, 本文选取的样本数据 和环境数据从时间分布上来说基本一致，因此该环 境数据和天麻样本点数据可以用来预测天麻的分
布。一般来说, 非生物的因素(主要是气候因素)主要 在大尺度空间影响物种的分布，因此本文主要选取 气候因子作为环境数据来预测天麻的分布。

\section{1 模型预测结果比较及准确性评估}

这3种模型的预测结果具有一定的相似性和差 异性。三种模型的预测结果基本一致：天麻全球潜 在适生区主要分布在 $20^{\circ}-50^{\circ} \mathrm{N}$ 的亚洲地区，其中 中国、日本和韩国是集中分布地。中国潜在适生区 区主要位于四川盆地附近的省区以及中东部，韩国 www.plant-ecology.com 
表2 三个模型的AUC值和Kappa值

Table 2 The AUC and Kappa values of the three models

\begin{tabular}{|c|c|c|c|c|c|c|}
\hline \multirow{2}{*}{$\begin{array}{c}\text { 组数 } \\
\text { No. of groups }\end{array}$} & \multicolumn{3}{|c|}{ AUC } & \multicolumn{3}{|c|}{ Kappa } \\
\hline & BIOCLIM & DOMAIN & MAXENT & BIOCLIM & DOMAIN & MAXENT \\
\hline 1 & 0.914 & 0.954 & 0.979 & 0.700 & 0.829 & 0.670 \\
\hline 2 & 0.945 & 0.972 & 0.980 & 0.829 & 0.851 & 0.651 \\
\hline 3 & 0.926 & 0.974 & 0.979 & 0.795 & 0.859 & 0.717 \\
\hline 4 & 0.902 & 0.943 & 0.985 & 0.759 & 0.829 & 0.637 \\
\hline 5 & 0.948 & 0.966 & 0.979 & 0.819 & 0.822 & 0.575 \\
\hline 6 & 0.960 & 0.967 & 0.981 & 0.833 & 0.806 & 0.664 \\
\hline 7 & 0.938 & 0.953 & 0.981 & 0.789 & 0.827 & 0.757 \\
\hline 8 & 0.940 & 0.962 & 0.980 & 0.806 & 0.883 & 0.816 \\
\hline 9 & 0.935 & 0.955 & 0.980 & 0.780 & 0.819 & 0.664 \\
\hline 10 & 0.949 & 0.963 & 0.977 & 0.800 & 0.851 & 0.790 \\
\hline
\end{tabular}

AUC, 受试者工作特征曲线下的面积; BIOCLIM, BIOCLIM模型; DOMAIN，域模型; MAXENT，最大熵模型。

AUC, area under receiver operating characteristic curve; BIOCLIM, BIOCLIM model; DOMAIN, domain model; MAXENT, maximum entropy model.

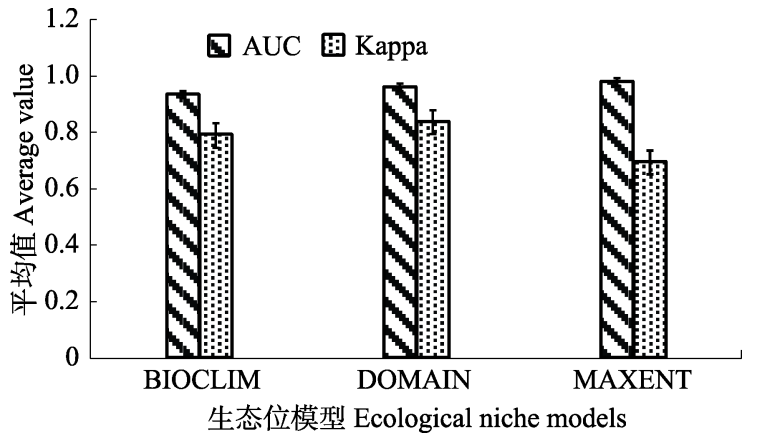

图2 3种生态位模型AUC和Kappa的平均值。AUC, 受试者 工作特征曲线下的面积; BIOCLIM，BIOCLIM模型；DOMAIN，域模型; MAXENT，最大熵模型。

Fig. 2 Average values of AUC and Kappa for the three ecological niche models. AUC, area under receiver operating characteristic curve; BIOCLIM, BIOCLIM model; DOMAIN, domain model; MAXENT, maximum entropy model.

潜在适生区主要位于韩国中东部的忠清北道、庆尚 北道、庆尚南道，日本潜在适生区主要位于本州岛、 九州岛以及四国岛地区。据文献报道, 中国天麻产 量高的地区主要分布在四川、贵州一带, 世界上出 产天麻的主要是亚洲东部的中国、韩国和日本(肖薇 等, 2011), 天麻的实际产区与本文3种模型预测的 适生区较一致，因此根据本文预测结果，亚洲可以 作为天麻的主要生产区, 而中国、韩国和日本是主 要发展地。但不同模型因原理不同, 结果也存在一 些差异, MAXENT和BIOCLIM模型预测的适生区分 布比较相似, 最适宜区范围也基本一致, 但 MAXENT模型比BIOCLIM模型预测结果范围要广, 因为 BIOCLIM 模型是基于环境包络原理, 而 MAXENT模型的原理是在给定的生态位条件下物 种分布达到摘值最大的状态。DOMAIN模型的预测
结果显示俄罗斯远东地区有部分适生区, 而且最适 宜区跨度较广, 这是因为DOMAIN模型是根据点与 点的相似矩阵进行分类, 受取样点影响较大, 有分 布点的地方几乎都能出现一定面积的适生区，所以 在俄罗斯地区, 由于存在分布点而出现一定面积的 适宜区。因此, 不同的模型具有自己的优缺点, 可以 相互参照, 综合比较, 选择一个合适的预测结果。

ROC和Kappa统计量是评价模型预测精度的两 种方法, 在物种分布模型评价中均得到了广泛的应 用(王娟和倪健, 2009; 张雷等, 2011), 因此本文选 择这两种方法来判断模型精度。本文采用 10 组数据 进行模型训练并采用相应的 10 组测试数据进行验证, 结果表明 3 种模型一致性显著, 预测精度较高, 比较 稳定可靠。因此 3 个模型均可用来预测天麻的潜在分 布区。相比较而言, MAXENT模型表现出更好的效 果, 其AUC平均值最大, 尽管其Kappa平均值相对 小一些，但是Kappa统计量是系列阈值判别能力的 最大值, 反映的是点判别能力, 而AUC值反映的是 系列阈值判别能力的综合值。模型在某一阈值的判 别能力强并不表示在其他阈值的判别能力也一定强, AUC值因不受判断阈值影响, 可更好地用于同一物 种不同模型的比较研究(王运生等, 2007)。

\section{2 天麻全球潜在适生区气候特征}

从分布区的地理环境来看, 天麻的适生区具有 一些共同的特点：均位于温带，气候类型属于温带 季风气候, 夏季高温多雨, 冬季寒冷干燥, 地势西 高东低使冬季季风得以加强(戴君虎等, 2013)。在全 球的其他地区，如北美洲、欧洲等，适宜性相对较差， 其整体气候与亚洲地区也有所差异, 表明天麻适宜 
于温带季风气候。从全球的地形地势来看, 天麻的 全球潜在适生区主要分布在中国四川盆地附近省份 和中东部，韩国中东部太白山脉、庆尚山脉、小白 山脉附近以及日本乌拉尔山脉附近, 这种地形地势 与温带季风气候的形成也有着密不可分的关系。最 适宜区主要分布在亚洲中部，向北和向南均分布较 少或适宜性相对较差, 说明天麻分布带虽然较广, 但最适宜生长的分布区相对集中, 因此接下来着重 研究天麻集中分布区的小范围生境有助于揭示天麻 的生态特征(石子为等, 2016)。研究表明在实际栽培 过程中, 土壤与天麻的生长及质量也息息相关, 如 土壤中矿质元素、微生物含量、有机质含量等因素 也影响天麻生长(刘洋等, 2007), 由于气候在大尺度 上控制物种的分布(周广胜和王玉辉, 2003), 本文根 据气候因子在全球范围内进行预测, 但在小范围内 土壤因素也会影响天麻生长, 因此预测某一局部地 区时，应适当兼顾土壤、地形、土地利用等多重因 素, 以更好地为天麻的引种栽培提供精细指导。

\section{3 天麻全球潜在适生区与天麻质量}

天麻作为一种药用植物, 其产量与质量并重, 目前的物种分布模型有利于大尺度地预测天麻潜在 分布区, 可为扩大天麻生产面积以及人工栽培提供 参考, 避免盲目引种造成的人力物力浪费和损失; 也有利于探寻野生天麻资源分布和保护天麻资源。 物种分布模型是基于天麻样本点及其生长区环境参 数进行预测, 理论上来说, 预测的天麻适宜分布区 与天麻品质有一定的联系, 因为收集的天麻样本点 主要是在天麻的已知产区, 天麻品质相对较好, 采 用生态位模型分析得到其潜在适生区，根据气候相 似性原理(魏淑秋, 1984), 潜在适生区的生态位环境 与原产地较为相似, 因此潜在分布区内的天麻品质 和原产区品质存在一定程度的相似性。由于本研究 没有进一步进行化学品质评价, 没有数据明确表明 适宜分布区和天麻品质好的地区完全等同。本文结 果显示最适宜区实际分布的天麻产量高, 品质相对 较好, 这表明适宜的环境有利于植物生长, 有更大 的可能性生产出高品质药材, 所以预测结果也可为 天麻质量分布提供参考。

基金项目 国家自然科学基金(81473304)和国家科 技支撑计划项目(2015BAI05B01)。

\section{参考文献}

Busby JR (1991). BIOCLIM - A bioclimate analysis and prediction system. Plant Protection Quarterly, 6, 64-68.

Carpenter G, Gillison AN, Winter J (1993). DOMAIN: A flexible modelling procedure for mapping potential distributions of plants and animals. Biodiversity \& Conservation, $2,667-680$.

Chai K, Liu HC, Li JL, Luo FL, Wang HL, Huang MJ, Luo CL, Zhao Z (2014). Genetic diversity of Gastrodia elata based on SRAP analysis. Chinese Traditional and Herbal Drugs, 45, 2974-2981. (in Chinese with English abstract) [柴锟, 刘红昌，李金玲，罗夫来，王华否，黄明进，罗春丽，赵 致 (2014). 基于SRAP分子标记的天麻遗传多样性研究. 中草药, 45, 2974-2981.]

Dai JH, Wang HJ, Ge QS (2013). Changes of spring frost risks during the flowering period of woody plants in temperate monsoon area of China over the past 50 years. Acta Geographica Sinica, 68, 593-601. (in Chinese with English abstract) [戴君虎, 王焕炣, 葛全胜 (2013). 近50年 中国温带季风区植物花期春季霜冻风险变化. 地理学 报, 68, 593-601.]

Engler R, Randin CF, Thuiller W, Dullinger S, Zimmermann NE, Araújo MB, Pearman PB, Le Lay G, Piedallu C, Albert $\mathrm{CH}$, Choler P, Coldea G, de Lamo X, Dirnböck T, Gégout JC, Gómez-García D, Grytnes JA, Heegaard E, Høistad F, Nogués-Bravo D, Normand S, Puşcaş M, Sebastià MT, Stanisci A, Theurillat JP, Trivedi R, Vittoz P, Guisan A (2011). 21st century climate change threatens mountain flora unequally across Europe. Global Change Biology, 17, 2330-2341.

Graham MH (2003). Confronting multicollinearity in ecological multiple regression. Ecology, 84, 2809-2815.

$\mathrm{Hu}$ RL (2015). Analysis of cultivation and management technical of Gastrodia elata. Agriculture \& Technology, 35(4), 111. (in Chinese with English abstract) [胡荣丽 (2015). 天麻栽培管理技术分析. 农业与技术, 35(4), 111.]

Hu YB, Cui J, Han X, Qiu DW, Xu JY (2001). Research progress of Gastrodia elata. Journal of Guiyang Medical College, 23(4), 48-51. (in Chinese with English abstract) [胡一冰, 崔佳, 韩笑, 邱德文, 许建阳 (2001). 中药天 麻研究进展. 贵阳中医学院学报, 23(4), 48-51.]

Kim HJ, Lee SR, Moon KD (2003). Ether fraction of methanol extracts of Gastrodia elata, medicinal herb protects against neuronal cell damage after Transient Global Ischemia in Gerbils. Phytotherapy Research, 17, 909-912.

Kulhanek SA, Leung B, Ricciardi A (2011). Using ecological niche models to predict the abundance and impact of invasive species: Application to the common carp. Ecological Applications, 21, 203-213.

Liu Y, Zhang ZS, He YL, Zhang BG, Li XA (2007). Quality of crude traditional Chinese drugs and ecological environment.

www.plant-ecology.com 
World Science and Technology / Modernization of Traditional Chinese Medicine and Materia Medica, 9(1), 65-69. (in Chinese with English abstract) [刘洋, 张佐双, 贺玉 林, 张本刚, 李先恩 (2007). 药材品质与生态因子关系 的研究进展. 世界科学技术-中医药现代化, 9(1), 65-69.]

Manel S, Williams HC, Ormerod SJ (2001). Evaluating presence-absence models in ecology: The need to account for prevalence. Journal of Applied Ecology, 38, 921-931.

Peng HS, Wang DQ (2007). Affecting ecological factor on location of Gastrodia elata. Research and Practice of Chinese Medicines, 22(2), 6-9. (in Chinese with English abstract) [彭华胜, 王德群 (2007). 生态因子与古今天 麻产区的关系. 现代中药研究与实践, 22(2), 6-9.]

Peterson AT, Monica P, Eaton M (2007). Transferability and model evaluation in ecological niche modeling: A comparison of GARP and Maxent. Ecography, 30, 550-560.

Peterson AT, Soberón J, Pearson RG, Anderson RP, Nakamura M, Martinez-Meyer E, Araújo MB (2011). Ecological Niches and Geographical Distributions. Princeton University Press, Princeton, USA.

Phillips SJ, Anderson RP, Schapire RE (2006). Maximum entropy modeling of species geographic distributions. Ecological Modelling, 190, 231-259.

Segurado P, Araújo MB (2004). An evaluation of methods for modelling species distributions. Journal of Biogeography, $31,1555-1568$.

Shao P, Zeng XD (2011). The impact of interannual climate variability on the mean global vegetation distribution. Acta Ecologica Sinica, 31, 1494-1505. (in Chinese with English abstract) [邵璞, 曾晓东 (2011). 气候年际变率对全 球植被平均分布的影响. 生态学报, 31, 1494-1505.]

Shi ZW, Ma CJ, Kang CZ, Wang L, Zhang ZH, Chen JF, Zhang XB, Liu DH (2016). Ecological suitability regionalization for Gastrodia elata in Zhaotong based on Maxent and ArcGIS. China Journal of Chinese Materia Medica, 41, 3155-3163. (in Chinese with English abstract) [石子 为, 马聪吉, 康传志, 王丽, 张智慧, 陈骏飞, 张小波, 刘大会 (2016). 基于空间分析的昭通天麻生态适宜性 区划研究. 中国中药杂志, 41, 3155-3163.]

State Pharmacopoeia Commission (2015). People's Republic of China Pharmacopoeia ( I ). Chinese Medical Science and Technology Press, Beijing. 39. (in Chinese) [国家药典委 员会 (2015). 中华人民共和国药典 (一部). 中国医药科 技出版社，北京. 58.]

Sun LF, Zhang YH, Yang GT, Qin GF, Song YS, Song RQ, Zhao J, Tian SM (2007). Geographic distribution survey of Armillaria biological species in Heilongjiang Province. Mycosystema, 26, 59-67. (in Chinese with English abstract) [孙立夫, 张艳华, 杨国亭, 秦国夫, 宋玉双, 宋瑞清, 赵俊, 田淑敏 (2007). 黑龙江省蜜环菌生物种的地理分 布概况. 菌物学报, 26, 59-67.]
Swets JA (1988). Measuring the accuracy of diagnostic systems. Science, 240, 1285-1293.

Tao YH (2008). Recent progress on pharmacological effects of Gastrodia elata. China Journal of Chinese Materia Medica, 33, 108-110. (in Chinese with English abstract) [陶云 海 (2008). 天麻药理研究新进展. 中国中药杂志, 33, 108-110.]

Wang J, Ni J (2009). Modelling the distribution of five Caragana species in temperate northern China. Chinese Journal of Plant Ecology, 33, 12-24. (in Chinese with English abstract) [王娟, 倪健 (2009). 中国北方温带地区 5 种锦 鸡儿植物的分布模拟. 植物生态学报, 33, 12-24.]

Wang LF, Zhou CX (2013). Management of cultivation of organic Gastrodia elata in Changbai Mountain. Agriculture \& Technology, 33(12), 114-117. (in Chinese with English abstract) [王丽馥, 周常霞 (2013). 长白山有机天麻栽 培管理浅析. 农业与技术, 33(12), 114-117.]

Wang YS (2007). Application of Niche Models in the Risk Assessment of Invasive Alien Species. PhD dissertation, Hunan Agricultural University, Changsha. 3-113. [王运生 (2007). 生态位模型在外来入侵物种风险评估中的应用 研究. 博士学位论文, 湖南农业大学, 长沙. 3-113.]

Wang YS, Xie BY, Wan FH, Xiao QM, Dai LY (2007). Application of ROC curve analysis in evaluating the performance of alien species' potential distribution models. Biodiversity Science, 15, 365-372. (in Chinese with English abstract) [王运生，谢丙炎，万方浩，肖启明，戴良英 (2007). ROC曲线分析在评价入侵物种分布模型中的应 用. 生物多样性, 15, 365-372.]

Wei SQ (1984). Brief of Introduction of Agrometeorological Similarity. Journal of China Agricultural University, 10, 427-428. (in Chinese) [魏淑秋 (1984). 《农业气候相似 距库》简介. 北京农业大学学报, 10, 427-428.]

Xiao W, Yin M, Zhuang ZH, Huang J, Chen ZF, He XD, Cao YN, Mao KC (2011). Determination of gastrodin content in Gastrodia elata from different origins. Beijing Journal of Traditional Chinese Medicine, 30, 945-946. (in Chinese with English abstract) [肖薇, 尹珢, 庄志宏, 黄键, 陈志 峰, 何晓东, 曹艳楠, 毛克臣 (2011). 不同产地天麻中 天麻素含量的测定. 北京中医药, 30, 945-946.]

Xu JT (2013). Review of cultivation history with 50 years of Gastrodia elata. Edible and Medicinal Mushrooms, 21, 58-63. (in Chinese with English abstract) [徐锦堂 (2013). 我国天麻栽培 50 年研究历史的回顾. 食药用菌, 21 , 58-63.]

Xu WN, Wang PX, Han P, Yan TL, Zhang SY (2011). Application of Kappa coefficient to accuracy assessments of drought forecasting model: A case study of Guanzhong Plain. Journal of Natural Disasters, 20(6), 81-86. (in Chinese with English abstract) [许文宁, 王鹏新, 韩萍, 严泰来, 张树誉 (2011). Kappa系数在干旱预测模型精 度评价中的应用一以关中平原的干旱预测为例. 自 
然灾害学报, 20(6), 81-86.]

Zhang JX, Gui Y, Yang TJ, Wang Q, Jin JF, Zhu GS (2013). Development status and counter measures of Gastrodia elata in Guizhou. Guizhou Agricultural Sciences, 41(12), 170-173. (in Chinese with English abstract) [张金霞, 桂 阳, 杨通静, 王沈, 金吉芬, 朱国胜 (2013). 贵州天麻 生产现状与发展对策. 贵州农业科学, 41(12), 170-173.]

Zhang L, Liu SR, Sun PS, Wang TL (2011). Comparative evaluation of multiple models of the effects of climate change on the potential distribution of Pinus massoniana. Chinese Journal of Plant Ecology, 35, 1091-1105. (in Chinese with English abstract) [张雷, 刘世荣, 孙鹏森, 王同立 (2011). 气候变化对马尾松潜在分布影响预估 的多模型比较. 植物生态学报, 35, 1091-1105.]

Zhang MG, Ferry Slik JW, Ma KP (2016). Using species dis- tribution modeling to delineate the botanical richness patterns and phytogeographical regions of China. Scientific Reports, 3, 1-9.

Zhou GS, Wang YH (2003). Global Ecology. Meteorological Press, Beijing. 119-122. (in Chinese) [周广胜, 王玉辉 (2003). 全球生态学. 气象出版社, 北京. 119-122.]

Zhu GP, Liu GQ, Bu WJ, Gao YB (2013). Ecological niche modeling and its applications in biodiversity conservation. Biodiversity Science, 21, 90-98. (in Chinese with English abstract) [朱耿平, 刘国卿, 卜文俊, 高玉葆 (2013). 生态位模型的基本原理及其在生物多样性保护 中的应用. 生物多样性, 21, 90-98.]

责任编委: 王志恒 责任编辑: 王 葳

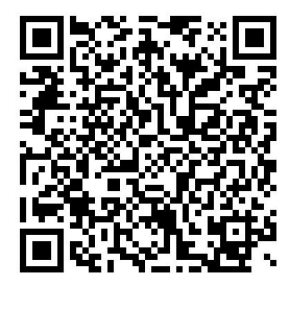

